\title{
Comparative prognostic value of radionuclide ventriculography at rest and during exercise in 100 patients after first myocardial infarction
}

\author{
N G DEWHURST, ^ A L MUIR \\ From the Department of Medicine, The Royal Infirmary, Edinburgh
}

SUMMARY To assess the prognostic value of radionuclide ventriculography we followed 100 consecutive patients for two years after their first myocardial infarction. Ventricular performance as assessed by left ventricular ejection fraction was measured at rest one day before hospital discharge and again one, four, and 12 months later at rest and during submaximal exercise. Left ventricular ejection fraction at discharge was below normal $(<0.47)$ in 74 patients and correlated well with abnormality of regional wall motion. Low left ventricular ejection fraction $(<0.30)$ observed in 25 patients was poorly predicted either clinically, radiologically, or electrocardiographically. Peak serum creatine kinase correlated well with left ventricular ejection fraction but there was a significant difference between anterior $(n=46)$ and inferior $(n=54)$ infarction with relative sparing of left ventricular function after inferior infarction. Left ventricular ejection fraction after inferior infarction was significantly higher both for transmural and subendocardial infarction, reflecting a variable degree of right ventricular necrosis.

Within two years of first infarction, angina had developed in 32 patients, left ventricular failure in 17, ventricular tachycardia or fibrillation in four patients, reinfarction in seven, and 17 died suddenly. Low resting left ventricular ejection fraction before discharge was associated with left ventricular failure, ventricular arrhythmia, and sudden death (10 patients). Of the remaining patients exercised one month after infarction 27 showed a significant fall $(>5 \%)$ in left ventricular ejection fraction and 23 of these developed postinfarction angina, four suffered non-fatal reinfarction and five patients died. Radionuclide ventriculography with exercise testing thus identified $88 \%$ of patients who died suddenly in the first two years after infarction. This was superior to any of the coronary prognostic indices currently in use and more predictive than the conventional end points of exercise testing (chest pain or ST segment depression) used either separately or in combination.

It has been shown that prognosis after myocardial infarction is directly related to the extent of left ventricular necrosis and to the function of the residual noninfarcted ventricle. ${ }^{1-3}$ The many clinical variables evident in the acute phase are a reflection of the degree of ventricular damage and correlate well with a noninvasive radionuclide assessment of ventricular function in the convalescent period. ${ }^{4-6}$ Recent studies, however, have failed to agree on the natural history of ventricular function over the ensuing year

^Present address: Department of Cardiology, The Royal Infirmary, Edinburgh.

Accepted for publication 12 October 1982
1478 and have not yet been compared with the traditional prognostic indices such as those used by Peel et al. ${ }^{9}$ Norris et al. ${ }^{10}$ and Luria et al. ${ }^{11}$ With the current interest in rehabilitation and early mobilisation, favourable results have been published on the ability of a submaximal exercise test to predict morbidity and mortality in patients leaving hospital ${ }^{12-14}$ Despite this initial optimism Borer et al. ${ }^{8}$ failed to confirm any relation between exercise induced ST segment depression and either mortality or mechanical function assessed by radionuclide ventriculography.

In the current study we have compared the results of radionuclide ventriculography with clinical, electrocardiographic, radiographic, and biochemical indices of infarct size, and compared this with traditional 
coronary prognostic indices. We have documented the natural history of ventricular function and compared the exercise induced changes in ejection fraction with standard exercise testing criteria in their ability to predict morbidity and mortality in 100 consecutive patients surviving to leave hospital after their first myocardial infarction.

\section{Subjects and methods}

We studied 100 consecutive patients ( 84 men and 16 women) who had been transferred to a general medical unit after variable length of stay in the coronary care unit (one to seven days, mean 2.3 days) after their first myocardial infarction. Patients over the age of 70 were excluded and the mean age was 57 (range 38 to 69 years). All patients satisfied the three criteria for acute myocardial infarction; myocardial ischaemic pain lasting more than 30 minutes, a rise in serum levels of two or three cardiac enzymes (aspartate aminotransferase, lactate dehydrogenase, or creatine kinase), and electrocardiographic evidence of acute infarction. Infarct location was characterised according to established electrocardiographic criteria as anterior (including anteroseptal and anterolateral) or inferior (including inferolateral and inferoposterior). The site of infarction was "inferior" in 54 and "anterior" in 46 patients. Infarction was transmural with the development of new $Q$ waves in 84 patients, and 16 had electrocardiographic changes confined to the ST segment or $\mathrm{T}$ wave compatible with subendocardial infarction. Of the 100 patients 75 were smokers at the time of admission and 12 were exsmokers, 17 had a history of angina of effort, 11 were taking antihypertensive drugs, and the two youngest women were taking oral contraceptive preparations. The only other non-cardiac conditions were seen in three patients with insulin independent diabetes mellitus, two with chronic obstructive airways disease, and three with a previous history of peptic ulcer.

Clinical progress was carefully monitored in the coronary care unit, and serious early arrhythmias defined as ventricular tachycardia, ventricular fibrillation, complete heart block, or asystole were recorded. The early progress of 25 patients was complicated by serious arrhythmias within 12 hours of the onset of pain. Thirteen patients suffered arrhythmia after inferior myocardial infarction (five ventricular tachycardia, five ventricular fibrillation, two complete heart block, one asystole) and 12 after anterior infarction (three ventricular tachycardia, nine ventricular fibrillation). Clinical signs of overt heart failure in the acute phase (tachycardia, gallop rhythm, and persistent crepitations) were noted. Left ventricular failure diagnosed radiologically as alveolar oedema was observed in 13 patients after inferior infarction and 25 patients after anterior infarction. Heart size was esti- $\stackrel{.}{.}$ mated by cardiothoracic ratio (CTR) method and $\vec{F}$. expressed as the ratio of the maximal cardiac diameter $\stackrel{?}{?}$ to the maximal intrathoracic diameter from a standard $\frac{}{2}$ six foot chest radiograph taken on the day before dis- $\frac{\bar{\sigma}}{\bar{D}}$ charge from hospital, values greater than 0.50 being $\frac{\vec{D}}{\vec{D}}$ considered to represent cardiomegaly. After transfer, $\stackrel{\mathbb{Q}}{\not}$ daily electrocardiograms were obtained and ventricu- is lar arrhythmias classified according to the modified $\overrightarrow{0}$ criteria of Lown. ${ }^{15}$ To characterise our patient population further the coronary prognostic indices of Peel $\vec{\omega}$ et al. ${ }^{9}$ Norris et al.,${ }^{10}$ and Luria et al. ${ }^{11}$ were calcu- $\odot$ lated for each patient.

Serum creatine kinase levels (CK) were obtained on $\overrightarrow{0}$ 92 patients admitted directly to the coronary care i unit. Venous blood was withdrawn on admission and $\vec{A}$ thereafter at 12 to 24 hour intervals until transfer. All $\vec{\circ}$ drugs were given intravenously and the timing, voltage, and frequency of any cardioversion procedure $\rightarrow$ noted. Total CK activity was measured using a mod- $\mathbb{\Phi}$ ification of the Rosalki technique. Serial enzyme val- $\frac{\bar{T}}{\mathrm{C}}$ ues were used to construct a CK profile for individual patients and a fractional decay rate of enzyme activity calculated if two or more CK levels had been meas- $\stackrel{\odot}{\circ}$ ured later than 24 hours after the onset of pain. $16 \mathrm{\omega}$ These data were available on 47 out of the 92 patients $\square$ admitted to the coronary care unit, and thus an aver- $\sum^{2}$ age value was calculated for the whole population and used to estimate a peak CK level 24 hours after the onset of pain.

\section{RADIONUCLIDE METHODS}

Isotope ventriculography was performed at rest on the $\frac{3}{5}$ day immediately before discharge from hospitalo (mean 11 days, range five to 19 days) when patients were well and clinically and radiologically clear of $\widetilde{\widehat{D}}$ heart failure. Subjects were informed of the nature of the investigation and had consented to take part. The radionuclide method used was that of gated blood: pool imaging, as described previously from thiso laboratory, and left ventricular ejection fraction was calculated from a corrected time activity curve. ${ }^{6}$ Left윽 ventricular wall motion was examined from a con- $\rightarrow$ tinuous movie display with and without edge enhancement methods. Abnormalities of regional walln movement were analysed by dividing the left ventricular wall into four segments of equal length, two lat -0 eral, one apical, and one septal. Dyskinetic segments were identified using a modified system of paradox imaging which displayed the change in volume signato by subtracting frame $n$ from frame $n+1$ and displayed areas of dyskinesis moving out of phase relative to the ${ }^{+}$ remainder of the ventricular wall. ${ }^{17}$ Low ejection frac $-\frac{T}{9}$ tion was frequently associated with ventricula extrasystoles and therefore a modified tape recordep was used to replay data to the computer and thळ 
ectopic and postectopic data rejected. ${ }^{18}$ Normal values for ejection fraction were measured in a group of 26 subjects of comparable age, with no history of cardiopulmonary disease.

\section{DRUG TREATMENT IN HOSPITAL}

Thirty-eight patients were treated with diuretics in the coronary care unit for acute phase heart failure and of these 21 remained on treatment throughout their stay in hospital. A further three patients were started on diuretics for heart failure after their stay in the coronary care unit, and therefore 24 patients were taking diuretics at the time of their predischarge ventriculogram. Twenty-two patients had been started on mexiletine because they had ventricular arrhythmias in the coronary care unit, and two were taking digoxin. At the time of predischarge ventriculography one patient was taking a long acting nitrate preparation and two were taking beta adrenoceptor antagonists.

\section{FOLLOW-UP PERIOD}

At one, four, and 12 months after acute infarction a clinical history was obtained, and physical examination, 12 lead electrocardiogram, and chest radiography performed. Repeat radionuclide ventriculography was performed at each occasion on all but three patients. One required surgical closure of a late ruptured interventricular septum, one developed atrial fibrillation after inferior infarction, and the other patient had moved from the area and could not be recalled at 12 months. In the remaining 97 patients exercise tolerance, exertional dyspnoea, and angina or any change in drugs were recorded. Antiarrhythmic treatment was discontinued routinely six weeks after infarction and diuretics reduced or stopped provided clinical progress and chest radiography were satisfactory. No therapeutic decisions were made on the basis of measurements of ejection fraction. A 24 hour ambulatory electrocardiographic tape recording was obtained if there was a history of either palpitation or syncope. No changes in drug treatment were made between hospital discharge and exercise testing at one month follow-up.

Progress was deemed to be uncomplicated or complicated by angina, reinfarction, heart failure, arrhythmia or sudden death, and death or survival confirmed for each patient without exception at the completion of the study. Death was attributed to coronary heart disease when an apparently well person was seen to collapse and die with no other cause of death suggested by history. Timing, circumstances, and certified cause of death were routinely requested from the patient's general practitioner.

\section{EXERCISE PROCEDURE}

At each follow-up appointment radionuclide ventriculography was repeated at rest and during exercise on a mechanically braked bicycle ergometer using a multistage symptom-limited exercise procedure. After the injection of $15 \mathrm{mCi}$ of technetium-99m labelled human serum albumin and acquisition of the resting data, exercise testing was performed in the supine, feet-up position. Exercise was started at a work load of $200 \mathrm{kilopond}-\mathrm{metres} / \mathrm{min}(\mathrm{kpm} / \mathrm{min})$ for women and $250 \mathrm{kpm} / \mathrm{min}$ for men. Instantaneous $R R$ interval was displayed and data collection begun after steady state had been achieved. Exercise was performed and data collection repeated over a range of increasing work load values $(200,400,600 \mathrm{kpm} / \mathrm{min}$ for women; $250,500,750 \mathrm{kpm} / \mathrm{min}$ for men) and between three and five minutes were spent at each work load. Electrocardiographic monitoring was performed during and for five minutes after exercise using standard chest lead V5 and cuff blood pressure monitored at one minute intervals. Exercise was terminated if the patient developed angina, severe fatigue, or dyspnoea, frequent $(>10 /$ minute) ventricular extrasystoles, or depression of the ST segment greater than $5 \mathrm{~mm}$. Patients who developed angina or ST segment depression were allowed to cornplete at least two minutes at that exercise level to permit collection of adequate scintigraphic data. In those patients with considerable variation in heart rate during any one exercise level the observer could select the RR interval containing the greatest proportion of cardiac cycles, thus permitting accurate estimation of exercise ejection fraction.

\section{STATISTICAL ANALYSIS}

The Wilcoxon rank sum and signed rank tests for non-parametric data were used in all statistical analysis. The F-distribution was used to test for coincidence of two regressions.

\section{Results}

RESTING STUDIES BEFORE DISCHARGE

Mean ejection fraction at rest was reduced for those patients recovering from acute myocardial infarction compared with normal subjects $(0.38 \pm 0.12$ versus $0.57 \pm 0.05$ mean $\pm S D)(p<0.01)$. The acute phase progress of 52 patients was totally uncomplicated, 25 had serious arrhythmia and 38 had left ventricular failure. Those patients with serious arrhythmia in the acute phase had low ejection fractions in the convalescent phase compared with those whose progress had been uncomplicated $(0.32 \pm 0.11$ versus $0.45 \pm 0.06$, $\mathrm{p}<0.01)$ (Fig. 1). Those patients with primary $(n=12)$ and complicating $(n=10)$ arrhythmias could not be distinguished by radionuclide ventriculography and had identical mean ejection fractions which were 


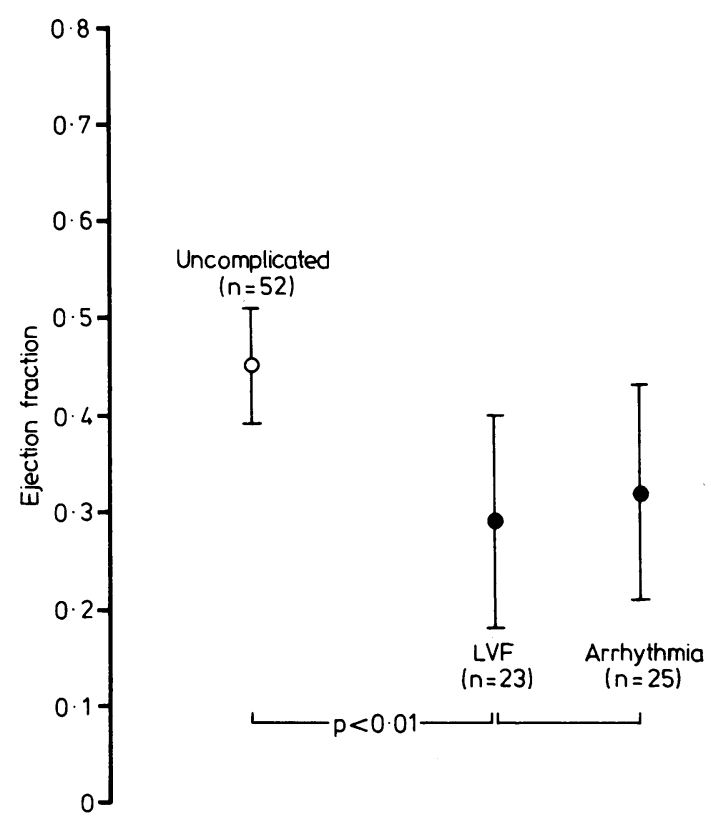

Fig. 1 Relation between left ventricular ejection fraction and early serious arrhythmia and left ventricular failure in the acute phase. Means ( $\pm S D$ ) are shown by the bars.

severely reduced (0.33). Patients with left ventricular failure in the acute phase, though clinically and radiologically clear of heart failure at the time of their first study, had a low ejection fraction in the convalescent phase $(0.29 \pm 0.11, \mathrm{p}<0.01)$. Transmural anterior myocardial infarction $(n=37)$ resulted in a greater reduction in ejection fraction than transmural inferior infarction $(n=46)(0.36 \pm 0.11$ versus $0.42 \pm 0.08$, $\mathrm{p}<0.01)$. The same difference was apparent for subendocardial infarction, anterior $(n=9)$ having significantly lower ejection fraction than inferior $(n=8)$ infarction $(0.42 \pm 0.08$ versus $0.53 \pm 0.04$, p $<0.01$ ) (Fig. 2).

Regional wall motion appeared normal in 27 patients and abnormal in 73, abnormalities corresponding to the site of infarction in 56. Segmental hypokinesis as the only abnormality was observed in 22 patients, hypokinesis affecting more than one segment was seen in 15 patients, and areas of akinesis were observed in 11 patients. Dyskinetic segments were seen in 25 patients (19 anterior infarction, six inferior infarction), all of whom had sustained the acute phase complications of either heart failure, serious arrhythmia, or both. Of these 18 had persisting ST segment elevation of $1 \mathrm{mV}$ or greater persisting for longer than 28 days, but this electrocardiographic feature was absent in four out of the six patients with inferolateral infarction. Similar ST segment elevation was observed in six patients who had no evidence of $C$ paradoxical wall motion. Global left ventricular per- $\overrightarrow{\vec{F}}$ formance correlated well with the presence of abnormalities of regional wall movement (Fig. 3). Mean ejection fraction associated with apparently normal $\frac{\bar{\sigma}}{\bar{N}}$ wall motion was $0.48 \pm 0.06$, with hypokinesis of more $\overrightarrow{\widetilde{\sigma}}$ than one segment it was $0.41 \pm 0.09$, with akinesis it 0 was $0.37 \pm 0.08$, and with dyskinesis it was $\%$ significantly reduced at $0.23 \pm 0.07(\mathrm{p}<0.001)$. $\overrightarrow{0}$

Excluding patients with a history of hypertension, radiological heart size correlated poorly with ejection $\vec{\omega}$ fraction $(r-0.29)$. Though ejection fraction was $\frac{\rho}{\partial}$ depressed in all patients with cardiomegaly, normal heart size was often associated with depressed left $\overrightarrow{0}$ ventricular function even when not clinically appar- ? ent. Likewise there was no significant correlation $\vec{\exists}$ between ejection fraction and the Norris coronary $\vec{\circ}$ prognostic index which includes radiological heart size as one of the predictors of subsequent mortality. $\vec{T}$

Left ventricular ejection fraction correlated well $\$$ with enzymatic indices of infarct size (Fig. $4 a$ and $4 b$ ). Peak serum CK levels were closely related to ejection $\stackrel{2}{2}$ fraction after anterior infarction $(r-0.63, p<0.001)$ but the relation was less obvious after inferior infarc- $\infty$ tion $(r-0.45, p<0.01)$. There was no significant difference between mean peak serum $\mathrm{CK}$ for anterior and inferior infarcts, and for an equivalent rise in

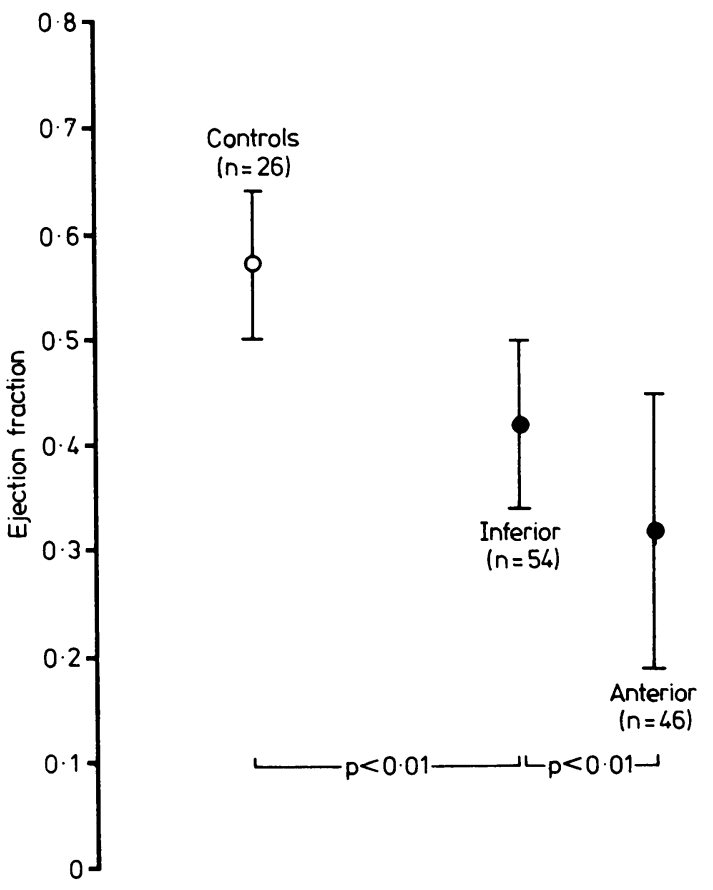

Fig. 2 Relation between left ventricular ejection fraction and site of infarction. Means $( \pm S D)$ are shown by the bars. 


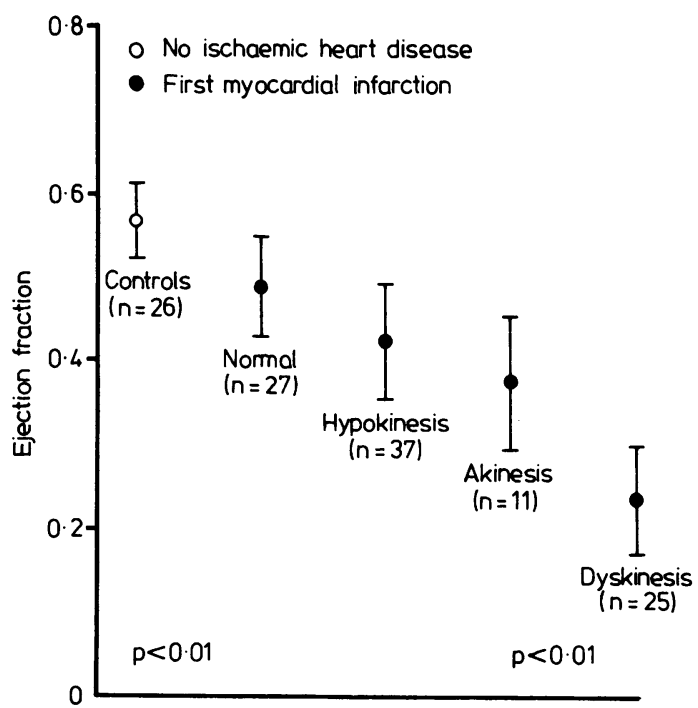

Fig. 3 Relation between left ventricular ejection fraction and regional wall motion abnormality. Means $( \pm S D)$ are shown by the bars.

serum CK levels there was significant sparing of left ventricular function after inferior infarction $(f=16.5$, $p<0.01$ ). Right ventricular enlargement and hypokinesis were seen in 23 patients all of whom had sustained inferior infarction.

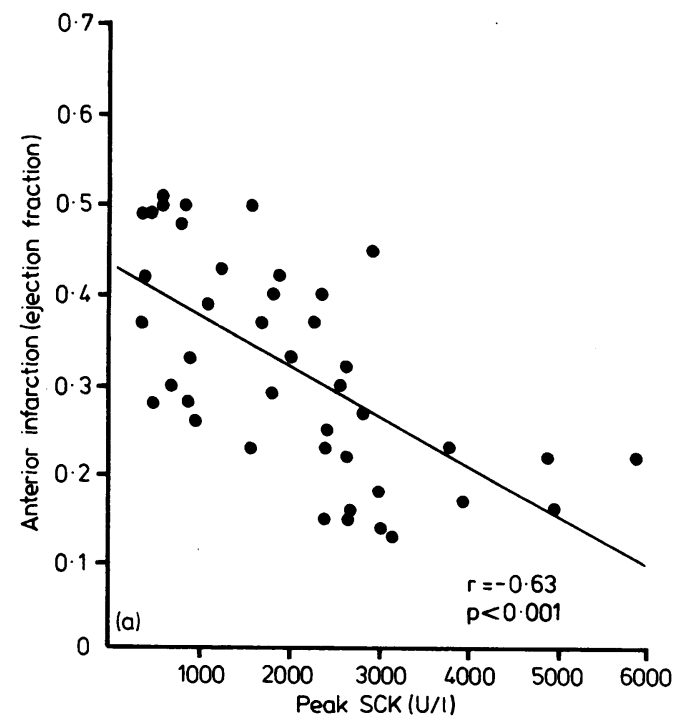

Fig. 4a, b Relation between left ventricular ejection fraction and peak serum creatine kinase (SCK) after anterior and inferior myocardial infarction.

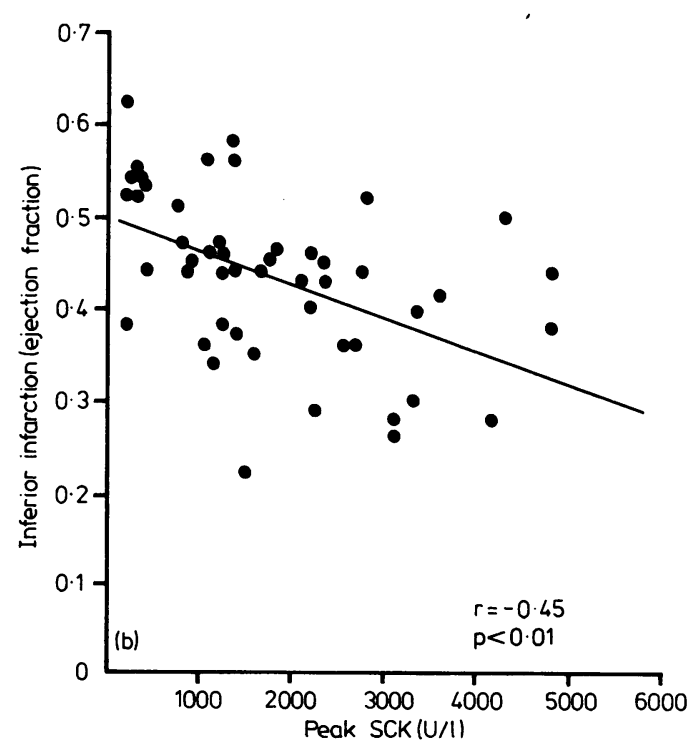

Of 69 patients who were in full-time employment before infarction 60 returned to work though 10 of these were re-employed in a less physically demanding capacity. Over the 12 month follow-up period 15 patients had documented episodes of left ventricular failure requiring emergency treatment by their general practitioner or readmission to hospital or both. Three additional patients developed signs of predominant right heart failure after transmural inferior myocardial infarction. Thirty-two patients developed post-infarction angina, six patients suffered non-fatal reinfarction, and two others had documented episodes of self-terminating ventricular tachycardia. There were no deaths in the first four weeks after discharge, four patients had died at four months, 11 at 12 months, and 17 at two years.

Low resting ejection fraction at discharge was associated with the development of left ventricular failure in the subsequent year. Without exception the 15 patients requiring maintenance or increased diuretic therapy had dyskinetic segments and severely reduced ejection fraction $(<0.35)$. Mean resting ejection fraction for the patients with initially normal wall motion or segmental hypokinesis did not change significantly over 12 months (Fig. 5) and for those with segmental akinesis ejection fraction increased from 0.37 to $0.45(p<0.05)$. The significant difference observed in ejection fraction after anterior and
RESTING STUDIES AT FOLLOW-UP 


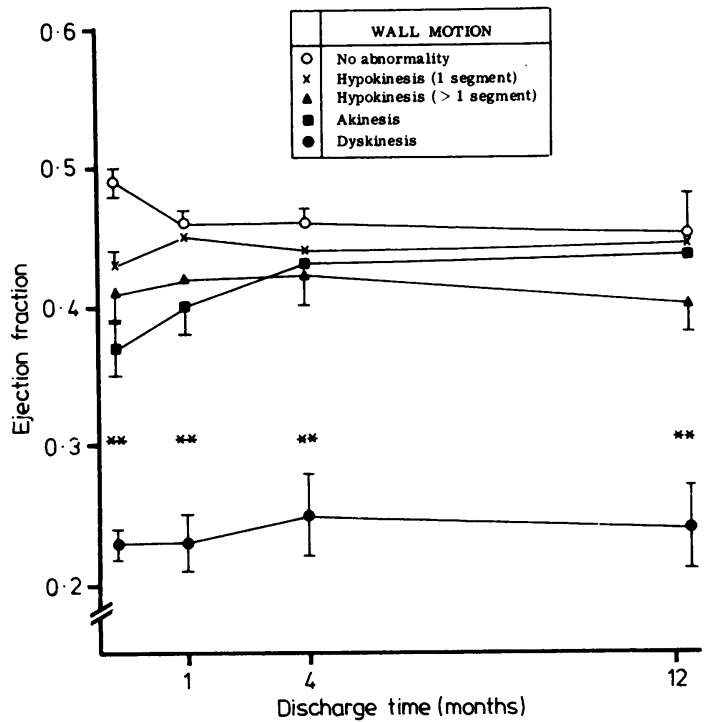

Fig. 5 Relation between initial abnormality of wall motion and changes in left ventricular ejection fraction after discharge from hospital. Means ( $\pm S E M)$ are shown by the bars. $\star \star=$ $p<0.01$.

inferior infarction before discharge persisted throughout the first 12 months (Fig. 6). This difference was the result of the excess incidence of ventricular dyskinesis and poor global left ventricular function after anterior infarction, since mean ejection fraction in this group failed to improve and remained unchanged at the end of one year $(0.23 \pm 0.07$ versus $0.24 \pm 0 \cdot 10$ ). Though in two patients small dyskinetic

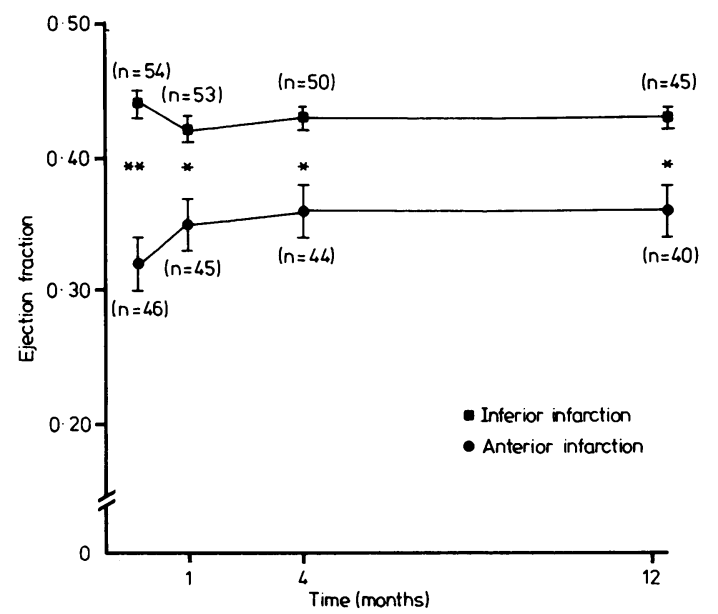

Fig. 6 Changes in left ventricular ejection fraction at rest after discharge from hospital. Means ( $\pm S E M$ ) are shown by the bars. $\star \star=p<0.01 ; \star=p<0.05$. segments resolved completely, the other patients had dyskinesis which persisted throughout the follow-up period.

There were 12 patients with frequent ventricular extrasystoles on the resting electrocardiogram before discharge from hospital. Complicated ventricular extrasystoles (Lown class III to V) were seen in seven patients, all of whom had dyskinetic areas, and two of these patients later developed documented episodes of ventricular tachycardia requiring readmission to hospital. Lown Class I to II ventricular extrasystoles were seen in five patients with either segmental akinesis or hypokinesis.

Of the 25 patients with dyskinetic areas and poor global function, $10(40 \%)$ with no other past medical history of note died within two years of discharge from hospital. These patients collapsed and died suddenly out of hospital and all but one died before medical attention could be summoned. Though myocardial infarction was the certified cause of death in all cases, only one patient gave a history of typical ischaemic pain. Of these 10 patients who died, seven were not predicted by either the Peel or Luria index and only three were deemed to be at high risk from the Norris index.

Left ventricular function, as assessed by resting ejection fraction, did not correlate with the patients' assessment of their own exercise tolerance or with return to previous employment. Similarly, frequency of angina pectoris did not correlate with resting ejection fraction.

\section{EXERCISE STUDIES AT ONE MONTH}

Two patients mentioned previously were excluded from repeat radionuclide ventriculography and a further two patients were not exercised because of frequent ventricular extrasystoles at rest. Of the remaining 96 patients, 94 were able to complete at least one level of exercise which was terminated prematurely in one because of frequent ventricular extrasystoles and in a second patient because of exercise related ventricular tachycardia. In these 94 patients the test was stopped because of angina in 12 and because of ventricular arrhythmias in three. ST segment depression of more than $1 \mathrm{~mm}$ in the absence of chest pain was observed in 11 and thus the test was deemed to be positive in a total of 28 patients.

In the 79 patients who did not develop angina or arrhythmia, exercise was terminated by either fatigue or dyspnoea. At the time of stopping the test 75 of these patients $(95 \%)$ had achieved a heart rate of $70 \%$ of the maximal heart rate predicted on the basis of age. Of the four patients with a limited heart rate response to exercise, two were taking beta adrenoceptor antagonists and the other two were young patients with slow resting heart rates. 
Twenty-six normal subjects subjected to an identical exercise procedure had a mean rise in ejection fraction of $14 \%$ of resting value, whereas patients recovering from myocardial infarction as a group were unable to increase ejection fraction significantly (mean fall2\%).

During submaximal exercise at one month, ejection fraction in patients with recent transmural anterior infarction fell significantly from $0.34 \pm 0.02$ to $0.30 \pm 0.02$ (mean change $=-11.5 \%$ of resting value, $p<0.01$, whereas for patients recovering from transmural inferior infarction there was no significant change $(0.49 \pm 0.02$ to $0.48 \pm 0.04)$. Those patients with poor left ventricular function and dyskinetic segments who completed at least one level of exercise $(n=20)$ had a fall in ejection fraction on exercise (mean fall - 6\%) secondary to an increase in paradoxical movement of the dyskinetic segment. They had been identified as a high risk group with poor prognosis on the resting study and the results of exercise testing were of no further value in predicting outcome. Of the remaining 74 patients there were 26 who were to develop post-infarction angina and none of these could increase ejection fraction on exercise (Fig. 7). Twenty-seven had a significant fall ( $>-5 \%)$ in ejection fraction and of these 21 developed postinfarction angina, two reinfarcted, and four died suddenly (Fig. 8). Six patients had already developed angina at the time of the exercise test one month after discharge; the remaining 15 were to develop angina in the subsequent 11 months. The operating characteristics of exercise ventriculography are shown in

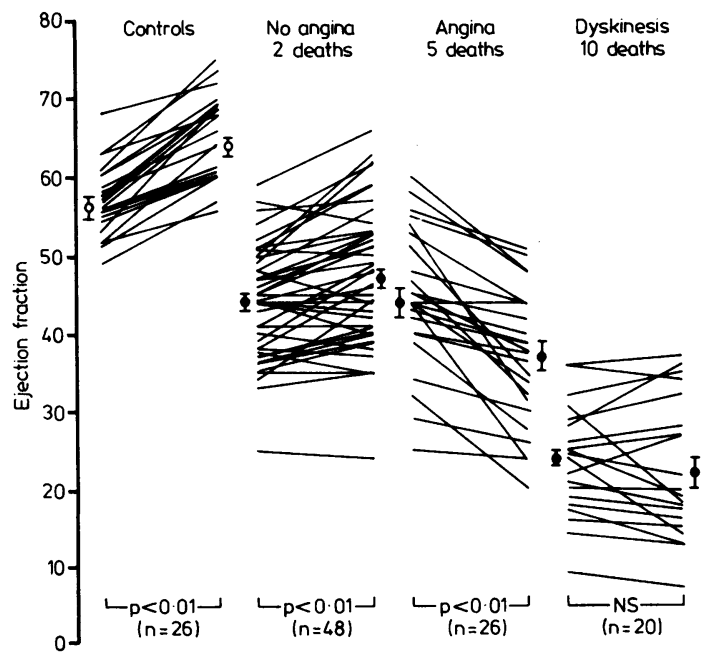

Fig. 7 Left ventricular ejection fraction at rest and during exercise one month after infarction. Changes related to morbidity and mortality in the following two years. Means ( \pm SEM) are shown by the bars.

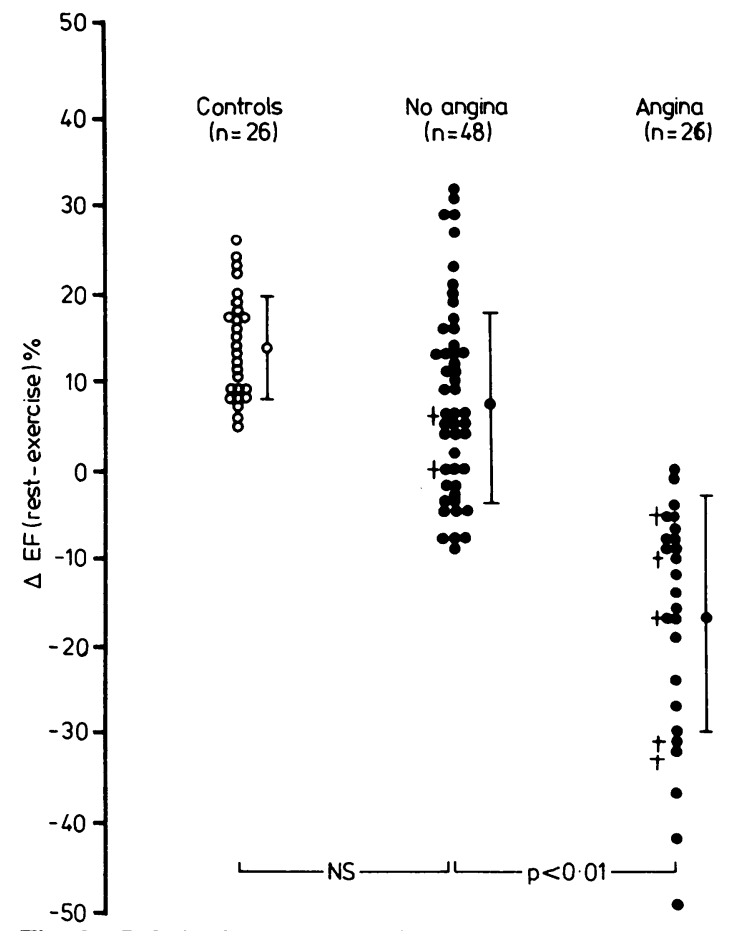

Fig. 8 Relation between change in ejection fraction on exercise and morbidity and mortality $(t)$. Means $( \pm S D)$ are shown by the bars.

Table 1. Of the four patients who died, three were identified as high risk by the Peel index and only one by the Norris index. The Luria index did not place any of the four patients as high risk survivors. Of the remaining 47 patients who showed little change in ejection fraction or were able to increase ejection fraction on exercise only three developed post-infarction angina and one of these sustained a further infarction. In this group there were two deaths that were sudden and were not predicted by either radionuclide ventriculography or any of the coronary prognostic indices employed.

Table 1 Operating characteristics of exercise radionuclide ventriculography in prediction of post-infarction angina

\begin{tabular}{|c|c|c|c|c|}
\hline & \multicolumn{4}{|l|}{ Angina } \\
\hline & Occurrence & & Non-occurrence & Total \\
\hline \multirow{3}{*}{$\begin{array}{l}\text { Ejection fraction } \\
\text { change }\end{array}$} & $\begin{array}{l}>-5 \% \\
+, 0\end{array}$ & 23 & 4 & 27 \\
\hline & $<-5 \%$ & 3 & 44 & 47 \\
\hline & Total & 26 & 48 & 74 \\
\hline
\end{tabular}

Sensitivity for subsequent post-infarction angina $=84 \%$. Specificity for subsequent post-infarction angina $=92 \%$.

False negative rate $\quad=12 \%$.

$\begin{array}{ll}\text { False positive rate } & =8 \% \text {. } \\ & =85 \% .\end{array}$ 
Using conventional end points of exercise testing (pain, ST segment depression $>1 \mathrm{~mm}$, or arrhythmia) there were 28 patients with positive tests of whom 15 developed angina and all of these had been predicted by the results of radionuclide exercise studies. There were a further 16 patients with negative tests who were to develop angina, and only one patient with significant ST depression died in the follow-up period. Five patients showed ST segment elevation during exercise and four of these had grossly abnormal wall motion (akinesis or dyskinesis) and though two of these patients had further infarcts, none died in the follow-up period. The operating characteristics of conventional exercise testing are shown in Table 2.

\section{EXERCISE STUDIES AT FOUR MONTHS}

Those patients who developed angina remained unable to increase ejection fraction on exercise at four months but the fall in ejection fraction in individual patients did not relate to the frequency of angina. For the majority of patients the results of exercise testing at one month were reproducible at four months and individual responses appeared consistent. There were, however, six patients who did not develop angina but gave a false positive result at one month, and all of these were subsequently able to increase ejection fraction on exercise at four months. Three patients having had initial increases in ejection fraction at one month converted to reduction in ejection fraction at four months though one of these subsequently reinfarcted, the other two have thus far remained well.

\section{MORTALITY}

Of the total of 17 deaths the Peel, Norris, and Luria indices predicted eight, four, and four, respectively, but all three indices were in agreement for only one patient. Nine patients died within two years of inferior infarction and four of these (44\%) had an ejection fraction of less than 0.35 . Of the eight patients who died after anterior infarction, seven $(88 \%)$ had poor left ventricular function, with ejection fraction

Table 2 Operating characteristics of the exercise test in prediction of post-infarction angina

\begin{tabular}{lllll}
\hline & Angina & & \\
\cline { 2 - 5 } & Occurrence & Non-occurrence & Total \\
\hline Exercise test & Positive & 15 & 13 & 28 \\
& Negative & 16 & 52 & 68 \\
& Total & 31 & 65 & 96 \\
\hline
\end{tabular}

Sensitivity for subsequent post-infarction angina $=48 \%$.

Specificity for subsequent post-infarction angina $=80 \%$.

False negative rate $\quad=52 \%$

False positive rate $\quad=20 \%$.

Predictive value less than 0.35 . Using resting ejection fraction less than 0.35 and a fall in ejection fraction on exercise greater than $5 \%$ radionuclide ventriculography predicted 15 of the 17 deaths.

\section{Discussion}

Prognosis after acute myocardial infarction has been shown to be dependent on a large number of variables all related directly or indirectly to the degree of myocardial necrosis and impairment of ventricular function..$^{1-3}$ The earliest studies were based purely on the clinical signs of extensive infarction such as heart failure, shock, persisting sinus tachycardia, and triple rhythm, but later radiological, electrocardiographic, and biochemical variables were taken into consideration. ${ }^{9-11}$ Indeed, with the use of such diverse methodology, prognosis can be determined at various points either on or during admission to the coronary care unit or alternatively immediately before the patient's discharge home. Authors therefore have independently developed coronary prognostic indices showing preferences for any one of the several variables included and in addition have used different statistical methods. Specific attempts at cross validation have been rare, but using their own patient population Bigger et al. ${ }^{19}$ were unsuccessful in an attempt to apply the set of variables previously shown by Moss et al. ${ }^{20}$ to be predictive of subsequent cardiac mortality. Because of these problems a more fundamental approach has been proposed and attempts have been made to relate prognosis not only to the extent of impairment of ventricular performance but also to the function of the residual noninfarcted myocardium. In a heterogeneous population of patients with ischaemic heart disease, the majority of whom had chronic stable angina, Hammermeister et al. ${ }^{21}$ proposed that left ventricular ejection fraction determined by contrast cineangiography was the single variable most predictive of survival. The use of noninvasive radionuclide techniques allows noninvasive measurement of left ventricular ejection fraction and prospective study of large groups of asymptomatic patients recovering from myocardial infarction.

\section{RESTING EJECTION FRACTION}

Acute phase progress complicated by either heart failure or serious arrhythmia correlated well with low ejection fraction just before discharge from hospital. The clinical relevance of pulmonary oedema in the acute phase of infarction has long been recognised, since many of these subjects suffer repeated episodes of heart failure or complicating arrhythmia in the convalescent phase. ${ }^{10-14}$ Despite the apparent wellbeing of patients before discharge many had a low ejection fraction not suspected clinically. As has been 
suggested by contrast ventriculography, radiological estimation of heart size is not an accurate predictor of ejection fraction (Field $e t a l .{ }^{22}$ ) and our own results confirm that there is no significant correlation between ejection fraction and cardiothoracic ratio. Though patients with cardiomegaly (CTR $>0.5)$ had reduced ejection fractions, low ejection fraction was frequently present with normal heart size determined radiologically. Likewise there was no correlation between ejection fraction and the Norris coronary prognostic index, which includes radiological heart size as one of the variables most predictive of survival. ${ }^{10}$

The prognostic implication of early ventricular arrhythmias in acute infarction is still uncertain. Although early work suggested that ventricular tachycardia and fibrillation had no adverse effects on long-term survival, ${ }^{23}$ more recently it has been suggested that arrhythmia in the acute phase is an adverse prognostic feature. ${ }^{24} 25$ From this study it is apparent that patients with primary and those with complicating arrhythmias cannot be distinguished by left ventricular performance, and in both groups mean ejection fraction is severely reduced. Enzymatic indices of infarct size can only be interpreted with caution after defibrillation, but from radionuclide assessment of global left ventricular function it is apparent that both groups had sustained extensive infarction.

Enzymatic indices of infarct size correlated well with global ejection fraction and though this has been shown previously with contrast ventriculography 26 thus far studies using radionuclide techniques have not been able to confirm this relation. ${ }^{8}$ As has been suggested by cineangiographic and haemodynamic studies of small populations there is a strong correlation between peak serum $\mathrm{CK}$ and variables of left ventricular function after anterior myocardial infarction. ${ }^{26}$ Despite equivalent peak levels of serum CK, however, we have confirmed the early observations of Sammel et al., ${ }^{27}$ that such a correlation is less pronounced after inferior myocardial infarction when there is significant sparing of left ventricular function. The most likely explanation for this striking difference is a variable degree of right ventricular necrosis, since right ventricular enlargement and hypokinesis were seen exclusively after inferior infarction and were observed in $43 \%$ of these patients.

There was a close relation between global left ventricular ejection fraction and the extent and severity of abnormality of regional left ventricular wall motion, the greatest reduction in ejection fraction being observed in those patients with areas of dyskinesis. Of all dyskinetic segments $80 \%$ followed anterior infarction and occurred in over half the patients recovering from transmural infarction. The preponderance of dyskinesis occurring after anterior infarction and a variable degree of right ventricular necrosis occurring after inferior infarction may be responsible for the striking differences seen in haemodynamic, cineangiographic, and radionuclide studies of the two groups. It has been shown that such differences are present within 24 hours of admission persisting throughout hospital stay,,$^{28}$ and from this study it is now evident that the difference is still apparent 12 months after discharge from hospital. In patients with akinetic or hypokinetic segments of left ventricular wall, global left ventricular function may improve significantly secondary to increased contractility of the residual viable myocardium, with stiffening of the infarcted area. In contrast, in the majority of patients extensive transmural infarction results in dyskinetic wall motion which persists over 12 months and is responsible for the poor global left ventricular function and limited left ventricular reserve which renders these patients at high risk of cardiac failure and sudden death during their convalescence. Persisting ST segment elevation, frequent complicated ventricular extrasystoles, and radiological cardiomegaly are only indirect indices of poor left ventricular function consequent upon dyskinesis, and as this study has shown are of limited value in the accurate prediction of high risk survivors from acute myocardial infarction.

Patients with low ejection fractions consequent upon extensive infarction were shown to have a high incidence of heart failure, complicated ventricular extrasystoles, or ventricular arrhythmia and sudden death in the first convalescent year. Of the six patients who were to die in the second year of follow-up, however, four had poor ventricular function and dyskinetic segments. Though the effect of infarct size on mortality has been said to be confined to the first few months of convalescence, ${ }^{29}$ it is evident that the associated excess incidence of sudden death may continue into the second postinfarction year.

\section{PREDICTIVE VALUE OF EXERCISE TESTING}

In the selected populations studied thus far it has been shown that exercise-induced ST segment depression and chest pain are predictive of subsequent cardiac events, whether exercise is performed before hospital discharge (Therroux et al. ${ }^{12}$ ) or three weeks after infarction (Sami et al. ${ }^{30}$ ). In the largest series of patients Théroux et al. ${ }^{12}$ reported increased frequency of angina and sudden death after observing ST segment depression during a submaximal treadmill test. Davidson and DeBusk ${ }^{13}$ confirmed these observations and in addition concluded that clinical characteristics and exercise induced ectopic activity were of no further predictive value. Despite these favourable results the limitations of electrocardiographic monitoring may be considerable and Borer et al. ${ }^{8}$ using a comparable submaximal treadmill test were unable to confirm the value of ST segment 
depression in predicting prognosis after myocardial infarction. Our own results suggest a greater predictive accuracy for ejection fraction measurements than electrocardiographic changes but were obtained using supine bicycle ergometry. Supine exercise has several technical advantages over upright exercise when performed in conjunction with gated blood pool imaging, but results in a different haemodynamic response and produces a higher systolic blood pressure and lower heart rate. Caldwell, ${ }^{31}$ however, in his comparison of the two modes of exercise found that in patients with coronary artery disease, despite these differences, the pressure-rate product was the same for both treadmill and supine bicycle exercise, and he concluded that the patients' limiting symptoms occurred at the same level of myocardial oxygen consumption. In the same study, the ST segment response was similar for the two positions of testing and it appears therefore that changes in heart rate do not alter the ability to detect ST segment shift.

The exercise procedure used in this study is very similar to that of Caldwell et al. ${ }^{31}$ and therefore the results we obtained using supine bicycle ergometry can justifiably be compared with the treadmill tests of Théroux et al. ${ }^{12}$ and Sami et al. ${ }^{30}$ The initial stress test was performed one month after discharge from hospital and since exercise tolerance improves spontaneously between one and four weeks after discharge (Markiewicz et al. ${ }^{32}$ ) the majority of patients were able to complete adequate work loads. Despite exercising in the supine position, of the 79 patients who did not experience angina or arrhythmia $95 \%$ achieved a heart rate of $70 \%$ of the maximal heart rate predicted on the basis of age. Using a supine exercise procedure, Bodenheimer et al. ${ }^{33}$ reported similar results and noted the superiority of ejection fraction changes to exercise induced ST segment depression in detecting the presence and severity of coronary artery disease.

The different functional ventricular response to exercise between patients with anterior and inferior infarction was noted by Pulido et al. ${ }^{34}$ who documented a fall in exercise ejection fraction after anterior infarction using supine bicycle ergometry. These results indicate, however, that this difference is the result of the excessive incidence of ventricular dyskinesis after anterior infarction since these patients with large dyskinetic segments as a group were unable to increase ejection fraction on exercise. Stress testing in this group may be hazardous and indeed is unnecessary since these patients have already been identified as high risk survivors from the resting study.

In the remaining patients with moderate or good left ventricular function we have shown that an early exercise test is a safe procedure and a fall in ejection fraction of more than $5 \%$ in the resting value is predictive of post-infarction angina and identifies a further group who are at risk of sudden death. Borer et al. ${ }^{8}$ were unable to correlate the results of exercise testing with prognosis but failed to exclude patients with poor left ventricular reserve and used a less stressful exercise procedure. Our own findings are consistent with those of Elkayam et al. ${ }^{35}$ who, studying 64 patients of whom 24 had a history of previous infarction, found that exercise induced changes in ejection fraction related closely to the number and severity of vessel stenoses determined by coronary angiography. Thus, it seems likely that radionuclide studies using a symptom limited exercise procedure can detect either multivessel disease or areas of potential ischaemia which result in the development of new regional wall dysfunction and an overall increase in end-systolic volume. Since, in the majority, ejection fraction changes were reproducible at four and 12 months, it seems probable that delay in developing symptoms of angina relates to the variability of individuals in the speed with which they return to either full mobility or employment.

Low ejection fraction is common after anterior myocardial infarction complicated in the acute phase by either left heart failure or serious arrhythmia, primary or complicating. Subsequent changes in ejection fraction both at rest and during exercise testing are predictive of the development of angina, left ventricular failure, and sudden death. As a single variable ejection fraction correlates well with all other variables directly related to infarct size and is therefore preferable to more indirect methods in risk stratification either to follow the natural history of coronary artery disease or assess the value of any therapeutic intervention.

\section{References}

1 Rahimtoola SH, Digilio MM, Ehsani A, Loeb HS, Rosen KM, Gunnar RM. Changes in left ventricular performance from early after acute myocardial infarction to the convalescent phase. Circulation 1972; 46: 770-9.

2 Norris RM, Caughey DE, Mercer CJ, Scott PJ. Prognosis after myocardial infarction. Six-year follow-up. $B r$ Heart $\mathcal{F}$ 1974; 36: 786-90.

3 Sobel BE, Bresnahan GF, Shell WE, Yoder RD. Estimation of infarct size in man and its relation to prognosis. Circulation 1972; 46: 640-8.

4 Rigo P, Murray M, Strauss HW, et al. Left ventricular function in acute myocardial infarction evaluated by gated scintiphotography. Circulation 1974; 50: 678-84.

5 Schelbert HR, Verba JW, Johnson AD, et al. Nontraumatic determination of left ventricular ejection fraction by radionuclide angiocardiography. Circulation 1975; 51: 902-9. 
6 Dewhurst NG, Hannan WJ, Muir AL. Prognostic value of radionuclide ventriculography after myocardial infarction. Qf Med 1980; 49: 479-90.

7 Rodger JC, Railton R, Young KC, Lakhani AK, Ghouri MAK. Nuclear angiography in convalescent phase of myocardial infarction. Serial study of left ventricular performance. Br Heart $\mathcal{F}$ 1980; 43: 499-505.

8 Borer JS, Rosing DR, Miller RH, et al. Natural history of left ventricular function during 1 year after acute myocardial infarction: comparison with clinical, electrocardiographic and biochemical determinations. Am $\mathcal{F}$ Cardiol 1980; 46: 1-12.

9 Peel AAF, Semple T, Wang I, Lancaster WM, Dall JLG. A coronary prognostic index for grading the severity of infarction. Br Hean $\mathcal{F}$ 1962; 24: 745-60.

10 Norris RM, Brandt PWT, Caughey DE, Lee AJ, Scott PJ. A new coronary prognostic index. Lancet 1969; i: 274-8.

11 Luria MH, Knoke JD, Wachs JS, Luria MA. Survival after recovery from acute myocardial infarction. Two and five year prognostic indices. Am $\mathcal{F}$ Med 1979; 67: 7-14.

12 Théroux P, Waters DD, Halpen C, Debaisieux JC, Mizgala HF. Prognostic value of exercise testing soon after myocardial infarction. $N$ Engl $\mathcal{F}$ Med 1979; 301: 341-5.

13 Davidson DM, DeBusk RF. Prognostic value of a single exercise test 3 weeks after uncomplicated myocardial infarction. Circulation 1980; 61: 236-42.

14 Jelinek VM, McDonald IG, Ryan WF, Ziffer RW, Clemens A, Gerloff J. Assessment of cardiac risk 10 days after uncomplicated myocardial infarction. $\mathrm{Br} \mathrm{Med} \mathcal{J}$ 1982; 284: 227-30.

15 Lown B. Sudden cardiac death: the major challenge confronting contemporary cardiology. Am f Cardiol 1979; 43: 313-28.

16 Norris RM, Whitlock RML, Barratt-Boyes C, Small CW. Clinical measurement of myocardial infarct size. Modification of a method for the estimation of total creatine phosphokinase release after myocardial infarction. Circulation 1975; 51: 614-20.

17 Dewhurst NG, Hannan WJ, Brash HM, Wraith PK, Muir AL. The prevalence and prognosis of ventricular dyskinesis after myocardial infarction using radionuclide ventriculography. Eur Heart f 1981; 2: 409-17.

18 Brash HM, Wraith PK, Hannan WJ, Dewhurst NG, Muir AL. The influence of ectopic heart beats in gated ventricular blood-pool studies. F Nucl Med 1980; 21: 391-3.

19 Bigger JT Jr, Heller CA, Wenger TL, Weld FM. Risk stratification after acute myocardial infarction. Am $\mathcal{J}$ Cardiol 1978; 42: 202-10.

20 Moss AJ, DeCamilla J, Davis H, Bayer L. The early posthospital phase of myocardial infarction. Circulation 1976; 54: 58-64.

21 Hammermeister KE, DeRouen TA, Dodge HT. Variables predictive of survival in patients with coronary disease. Circulation 1979; 59: 421-30.

22 Feild BJ, Russell RO Jr, Moraski RE, et al. Left ventricular size and function and heart size in the year following myocardial infarction. Circulation 1974; 50: 33139.

23 Lawrie DM. Long-term survival after ventricular fibril- lation complicating acute myocardial infarction. Lancet 1969; ii: 1085-7.

24 Denborough MA, Lovell RRH, Nestel PJ, Goble AJ. Arrhythmias and late sudden death after myocardial infarction. Lancet 1968; i: 386-8.

25 Schulze RA Jr, Strauss HW, Pitt B. Sudden death in the year following myocardial infarction. Relation to ventricular premature contractions in the late hospital phase and left ventricular ejection fraction. Am $\mathcal{Y}$ Med 1977; 62: 192-9.

26 Hori M, Inoue M, Fukui S et al. Correlation of ejection fraction and infarct size estimated from the total CK released in patients with acute myocardial infarction. $\mathrm{Br}$ Heart f 1979; 41: 433-40.

27 Sammel NL, Stuckey JG, Brandt PWT, Norris RM. Comparisons of enzymic with cineangiocardiographic estimations of myocardial infarct size. Br Heart $\mathcal{F}$ 1980; 43: 609-16.

28 Reduto LA, Berger HJ, Cohen LS, Gottschalk A, Zaret BL. Sequential radionuclide assessment of left and right ventricular performance after acute transmural myocardial infarction. Ann Intern Med 1978; 89: 441-7.

29 Geltman EM, Ehsani AA, Campbell MK, Schechtman $K$, Roberts $R$, Sobel BE. The influence of location and extent of myocardial infarction on long-term ventricular dysrhythmia and mortality. Circulation 1979; 60: 805-14.

30 Sami M, Kraemer H, DeBusk RF. The prognostic significance of serial exercise testing after myocardial infarction. Circulation 1979; 60: $1238-46$.

31 Caldwell JH, Hamilton GW, Sorensen SG, Ritchie JL, Williams DL, Kennedy JW. The detection of coronary artery disease with radionuclide techniques: a comparison of rest-exercise thallium imaging and ejection fraction response. Circulation 1980; 61: 610-9.

32 Markiewicz W, Houston N, DeBusk RF. Exercise testing soon after myocardial infarction. Circulation 1977; 56: 26-31.

33 Bodenheimer MM, Banka VS, Fooshee CM, Helfant RH. Comparative sensitivity of the exercise electrocardiogram, thallium imaging and stress radionuclide angiography to detect the presence and severity of coronary heart disease. Circulation 1979; 60: 1270-8.

34 Pulido JI, Doss J, Twieg G, et al. Submaximal exercise testing after acute myocardial infarction: myocardial scintigraphic and electrocardiographic observations. Am f Cardiol 1978; 42: 19-28.

35 Elkayam U, Weinstein M, Berman D, et al. Stress thallium-201 myocardial scintigraphy and exercise technetium ventriculography in the detection and location of chronic coronary artery disease: comparison of sensivity and specificity of these noninvasive tests alone and in combination. Am Heart $\mathcal{F}$ 1981; 101: 657-66.

Requests for reprints to Dr N G Dewhurst, Department of Cardiology, The Royal Infirmary, Edinburgh EH3 9YW. 